\title{
A NOTE ON UNIVERSALLY ZERO-DIVISOR RINGS
}

\author{
S. VISWESWARAN
}

\begin{abstract}
In this note we consider commutative rings with identity over which every unitary module is a zero-divisor module. We call such rings Universally Zero-divisor (UZD) rings. We show (1) a Noetherian ring $R$ is a UZD if and only if $R$ is semilocal and the Krull dimension of $R$ is at most one, (2) a Prüfer domain $R$ is a UZD if and only if $R$ has only a finite number of maximal ideals, and (3) if a ring $R$ has Noetherian spectrum and descending chain condition on prime ideals then $R$ is a UZD if and only if $\operatorname{Spec}(R)$ is a finite set. The question of ascent and descent of the property of a ring being a UZD with respect to integral extension of rings has also been answered.
\end{abstract}

\section{INTRODUCTION}

Let $R$ be a commutative ring with identity. Let $M$ be a unitary $R$-module. Recall that $M$ is said to be a Zero-divisor $R$-module if for every submodule $N$ of $M, N \neq M$, the set of zero divisors of $M / N$ (that is, $\{x \in R: x m \in N$ for some $m \in M \backslash N\}$ ) denoted by $Z_{R}(M / N)$ is the union of a finite number of prime ideals of $R, R$ is said to be a Zero-divisor ring (Z.D. ring) if $R$ is a Z.D. $R$-module [4]. In this note we study the properties of those commutative rings $R$ with identity for which every $R$-module is a Z.D. $R$-module.

All rings considered here are assumed to be commutative and with identity. If $A \subseteq B$ are rings we assume that $A$ and $B$ have the same identity element. By dimension of a ring we mean the Krull dimension. Modules are assumed to be unitary. Whenever a set $A$ is a subset of a set $B$ and $A \neq B$ we denote this symbolically as $A \subset B$.

We begin with the following definition.

We say a ring $R$ is a Universally Zero-divisor (UZD) ring if every $R$-module is a Z.D. $R$-module.

Received 5 April 1990

I am deeply indebted to Professor R. Raghavendran for his help, guidance and encouragement and I am also deeply indebted to Professor K.R. Nagarajan for his help and encouragement.

Copyright Clearance Centre, Inc. Serial-fee code: 0004-9729/91 \$A2.00+0.00. 
Proposition 1. Let $R$ be a ring. Then $R$ is a UZD if and only if the union of any family of prime ideals of $R$ is the union of a finite number of prime ideals of $R$ (not necessarily belonging to the same family).

Proof: Assume that $R$ is a UZD. Let $\left\{P_{\alpha}\right\}_{\alpha \in \Lambda}$ be any family of prime ideals of $R$. Let $M=\bigoplus_{\alpha \in A} R / P_{\alpha}$ (that is, direct sum of the $R$-modules $R / P_{\alpha}$ ), It is easy to see that $Z_{R}(M)=\bigcup_{\alpha \in \Lambda}^{\alpha \in \Lambda} P_{\alpha}$. Since $R$ is a UZD, $M$ is a Z.D. $R$-module and so $Z_{R}(M)=\bigcup_{i=1}^{t} Q_{i}$ for some finite number of prime ideals $Q_{1}, \ldots, Q_{t}$ of $R$. Thus $Z_{R}(M)=\bigcup_{\alpha \in \Lambda} P_{\alpha}=\bigcup_{i=1}^{t} Q_{i}$.

Conversely assume that the union of any family of prime ideals of $R$ is the union of a finite number of prime ideals of $R$. Let $M$ be any $R$-module. Let $N$ be a submodule of $M, N \neq M$. Notice that $R \backslash Z_{R}(M / N)$ is a saturated multiplicatively closed subset of $R$. Hence by $\left[1\right.$, Exercise 7 (i), p.44] $Z_{R}(M / N)$ is a union of prime ideals of $R$. By assumption it follows that $Z_{R}(M / N)$ is the union of a finite number of prime ideals of $R$. Thus $M$ is a Z.D. $R$-module. Hence we obtain that $R$ is a UZD.

REMARK 2. Using the above Proposition we see that $R$ is a UZD implies that any homomorphic image of $R$ is a UZD and $S^{-1} R$ is a UZD for every multiplicatively closed subset $S$ of $R, S \subseteq R \backslash\{0\}$.

Proposition 3. Let $R$ be an integral domain with quotient field $K$. Then $R$ is a UZD if and only if $K$ is a Z.D. $R$-module.

Proof: The "only if" part is clear. The "if" part follows from [13, Remark 2.1] and Proposition 1.

\section{Proposition 4.}

(i) Let $R$ be a Noetherian ring. Then $R$ is a UZD if and only if $R$ is semilocal and the dimension of $R$ is at most 1 .

(ii) A Prüfer domain $R$ is a UZD if and only if $R$ has only a finite number of maximal ideals.

The proof of Proposition 4 makes use of the following results.

LEMмa 5. If a ring $R$ is a UZD then $R$ has only a finite number of maximal ideals.

ProOf: Let $\left\{M_{\alpha}\right\}_{\alpha \in \Lambda}$ be the family of all maximal ideals of $R$. By Proposition 1, $\bigcup_{\alpha \in \Lambda} M_{\alpha}=\bigcup_{i=1}^{\infty} Q_{i}$ for some finite number of prime ideals $Q_{1}, \ldots, Q$, of $R$. Let $M_{i}(i=1, \ldots, s)$ be maximal ideals of $R$ such that $Q_{i} \subseteq M_{i}($ for $i=1, \ldots, s)$. Then it is clear that $\bigcup_{\alpha \in \Lambda} M_{\alpha}=\bigcup_{i=1}^{1} Q_{i}=\bigcup_{i=1}^{\infty} M_{i}$. It is now evident that distinct elements among $M_{1}, \ldots, M_{2}$ are all the maximal ideals of $R$. 
RESUlt 6. In a Noetherian ring every prime ideal has finite height [1, Corollary 11.12].

RESULT 7. In a Noetherian ring any prime ideal of height 2 contains an infinite number of height 1 prime ideals [11, Theorem 144].

Result 8. Let $I$ be any ideal of a Noetherian ring $R, I \neq R$. Then the set of prime ideals of $R$ which are minimal over $I$ is finite.

Result 8 follows by applying [1, Exercise 9, p.79] to the Noetherian ring $R / I$.

Proof of Proposition 4: (i) Assume that $R$ is a Noetherian ring and $R$ is a UZD. By Lemma $5, R$ is semilocal. We prove that the dimension of $R$ is at most 1 . Suppose that the dimension of $R$ is at least 2. Then by Result 6 it follows that there exists a prime ideal $p$ of $R$ such that height $p=2$.

Let $\left\{Q_{\alpha}\right\}_{\alpha \in \Lambda}$ be the set of all height one prime ideals of $R_{p}$. Note that $\left\{Q_{\alpha}\right\}_{\alpha \in \Lambda}=$ $\left\{P_{\alpha} R_{p}\right\}_{\alpha \in \Lambda}$ where $\left\{P_{\alpha}\right\}_{\alpha \in \Lambda}$ are prime ideals of $R$ such that height $P_{\alpha}=1$ and $P_{\alpha} \subset p$ for each $\alpha \in \Lambda$. By Result 7 it follows that $\Lambda$ is an infinite set. Result 8 and $[2$, Exercise 2, p.121] imply that there exists an element $y \in p R_{p}$ which is not in any of the minimal prime ideals of $\boldsymbol{R}_{\boldsymbol{p}}$. Now Result 8 implies that $y$ can belong to only a finite number of height 1 prime ideals of $R_{p}$. Let them be $\left\{P_{\alpha_{i}} R_{p}\right\}_{i=1}^{t}$. Let $A=\Lambda \backslash\left\{\alpha_{1}, \ldots, \alpha_{t}\right\}$. Then it is easy to see that $\bigcup_{\alpha \in A} P_{\alpha} R_{p}$ cannot be equal to the union of any finite number of prime ideals of $R_{p}$. This is in contradiction to the fact that $R_{p}$ is a UZD. Thus $R$ is semilocal and the dimension of $R$ is at most 1 .

Conversely if $R$ is semilocal and the dimension of $R$ is at most 1 then any prime ideal of $R$ is either a maximal ideal of $R$ or a minimal prime ideal of $R$. Since the set of minimal prime ideals of a Noetherian ring is finite we obtain that $R$ has only a finite number of prime ideals. It is then clear that $R$ is a UZD.

(ii) In view of Lemma 5, we need only prove the "if part" of (ii). Assume that $R$ is a Prüfer domain with only a finite number of maximal ideals $M_{1}, \ldots, M_{t}$. Let $\left\{P_{\alpha}\right\}_{\alpha \in \Lambda}$ be any family of prime ideals of $R$. Let $C_{i}$ be the union of those $P_{\alpha}$ 's which are contained in $M_{i}$ (for $i=1, \ldots, t$ ). Now $R_{M_{i}}$ is a valuation ring and so in the case $C_{i} \neq \emptyset, C_{i}$ is the union of some pairwise comparable prime ideals of $R$ and hence $C_{i} \in \operatorname{Spec}(R)$. This is true for $i=1, \ldots, t$. Further it is clear that $\bigcup_{\alpha \in \Lambda} P_{\alpha}=\bigcup_{i=1}^{t} C_{i}$
Hence by Proposition $1, R$ is a UZD. Remark 9. We have noted in Proposition 3 that an integral domain $R$ is a UZD if and only if the quotient field of $R$ is a Z.D. $R$-module. We now mention an example which shows (for an arbitrary ring $R$ ) that "the total quotient ring of $R$ is a Z.D. $R$ module" need not imply that $R$ is a UZD. Consider $T=Q(\sqrt{2})[[X, Y, Z]]$, the power series ring in three indeterminates $X, Y, Z$ over $Q(\sqrt{2})$ where $Q$ denotes the field of rationals. Let $M$ denote the unique maximal ideal of $T$. Let $S=Q+M$. Notice that 
the dimension of $T$ is 3 and $T$ is a finite integral extension of $S$. Hence by $[6,11.8$, p.106] and [3, Theorem 2] it follows that the dimension of $S$ is 3 and $S$ is Noetherian. Consider the chain of prime ideals (0) $\subset P_{1} \subset P_{2} \subset M$ of $S$ where $P_{1}=X T$ and $P_{2}=X T+Y T$. Let $R=S /(X S)$. We now show that the unique maximal ideal $M /(X S)$ of $R$ is full of zero divisors. For an element $m \in M$, let $m+X S \in M /(X S)$. Note that $(m+X S)(\sqrt{2} X+X S)=\sqrt{2}(m X)+X S=X(\sqrt{2} m)+X S=X S$ since $\sqrt{2} m \in M \subset S$. But $\sqrt{2} X \notin X S$. For if $\sqrt{2} X \in X S$ then we obtain $\sqrt{2} \in S$ which in turn implies that $\sqrt{2}=q+y$ for some $q \in Q, y \in M$. This implies that $\sqrt{2}-q=y \in Q(\sqrt{2}) \cap M=(0)$ and so $\sqrt{2}=q \in Q$ which is not true. Thus $\sqrt{2} X \notin X S$. This proves that $M /(X S)$ is full of zero divisors. Hence $R$ equals the total quotient ring of $R$. Since $R$ is a Noetherian ring, $R$ is a Z.D. $R$-module. Since the dimension of $R$ is 2, it follows from Proposition 4 (i) that $R$ is not a UZD.

Proposition 10. Let $R$ be a ring with Noetherian spectrum and descending chain condition on prime ideals. Then $R$ is a UZD if and only if $\operatorname{Spec}(R)$ is a finite set.

Proof: Assume that $R$ has Noetherian spectrum and has descending chain condition on prime ideals and $R$ is a UZD. The argument that we shall give below to show that $\operatorname{Spec}(R)$ is a finite set closely follows an argument of Heinzer and Lantz [10, Proposition 3.7]. By Lemma 5, $R$ has only a finite number of maximal ideals say $M_{1}, \ldots, M_{t}$. Let, if possible, $\operatorname{Spec}(R)$ be an infinite set. Then $\operatorname{Spec}\left(R_{M_{i}}\right)$ is an infinite set for some $i \in\{1, \ldots, t\}$. Now $R_{M_{i}}$ has Noetherian spectrum and so $M_{i} R_{M_{i}}=\sqrt{\left(y_{1}, \ldots, y_{h}\right) R_{M_{i}}}$ for some $y_{j} \in M_{i} R_{M_{i}}(j=1, \ldots, h)$ [12, Corollary 2.4]. It is then clear that $\operatorname{Spec}\left(R_{M_{i}}\left[1 / y_{j}\right]\right)$ is an infinite set for some $j \in\{1, \ldots, h\}$. Since $R_{M_{i}}\left[1 /\left(y_{j}\right)\right]$ is a UZD, it has only a finite number of maximal ideals say $N_{1}, \ldots N_{\varepsilon}$. Note that each $N_{g}(g=1, \ldots, s)$ is of the form $Q_{g} R_{M_{i}}\left[1 / y_{j}\right]$ for some prime ideal $Q_{g} R_{M_{i}}$ of $R_{M_{i}}$ such that $\mathrm{Q}_{g} R_{M_{i}} \subset M_{i} R_{M_{i}}$. Notice that $\operatorname{Spec}\left(\left(R_{M_{i}}\left[1 / y_{j}\right]\right)_{N_{g}}\right)$ is an infinite set for some $g \in\{1, \ldots, s\}$. Further observe that $M_{i} \supset \mathbf{Q}_{g}$. Now $\left(R_{M_{i}}\left[1 / y_{j}\right]\right)_{N_{g}} \simeq R_{\mathbf{Q}_{g}}$ by [2, Proposition 11 (iii), p.70] and thus $\operatorname{Spec}\left(R_{\mathbf{Q}_{g}}\right)$ is an infinite set and $R_{\mathbf{Q}_{g}}$ has Noetherian spectrum and is a UZD. Hence applying the above argument to the ring $R_{\mathbf{Q}_{g}}$ yields $H \in \operatorname{Spec}(R)$ such that $\mathbf{Q}_{g} \supset H$ and $\operatorname{Spec}\left(R_{H}\right)$ is infinite. So by repeating the above procedure we obtain a strictly descending sequence of prime ideals of $R$. This is in contradiction to the assumption that $R$ has descending chain condition on prime ideals. Therefore $\operatorname{Spec}(R)$ is a finite set.

The converse is obvious.

Remark 11. (i) We mention an example to show that the hypothesis in Proposition 10 that $R$ has Noetherian spectrum cannot be dropped. There exists a valuation ring $V$ such that the set of prime ideals of $V$ forms an infinite ascending chain (0) $\subset P_{1} \subset$ 
$P_{2} \subset \cdots \subset M=\bigcup_{i=1}^{\infty} P_{i}[5$, Example 5, p.578]. Thus $\operatorname{Spec}(V)$ is an infinite set but by Proposition 4 (ii), $V$ is a UZD. Further, note that $V$ has descending chain condition on prime ideals.

(ii) We now mention an example to show that the hypothesis in Proposition 10 that $\boldsymbol{R}$ has descending chain condition on prime ideals cannot be dropped.

Let $F$ be a field and $\left\{X_{i}\right\}_{i=1}^{\infty}$ be a set of elements algebraically independent over $F$. Let $K=F\left(\left\{X_{i}\right\}_{i=1}^{\infty}\right)$. Let $G$ be the direct sum of countably many copies of $Z$, the additive group of integers. We order $G$ with reverse lexicographic ordering. Then there exists a valuation ring $W$ on $K$ with value group $G$ by [7, Example 2.6]. It is easy to verify that the set of all prime ideals of $W$ forms an infinite descending chain $M \supset P_{1} \supset P_{2} \supset \ldots$ But $W$ is a UZD and $W$ has Noetherian spectrum.

Next we consider the ascent and descent of UZD with respect to integral extension of rings.

PROPOSITION 12. (i) Let $R \subset T$ be rings. Let $T$ be integral over $R$. If $T$ is a $U Z D$ then $R$ is a $U Z D$.

(ii) Let $B$ be a finite integral extension ring of a ring $A$. If $B$ has finitely many minimal prime ideals and if $A$ is a $U Z D$ then $B$ is a $U Z D$.

Proof: (i) Let $\left\{P_{\alpha}\right\}_{\alpha \in \Lambda}$ be any family of prime ideals of $R$. Now for each $P_{\alpha}$, there exists $Q_{\alpha} \in \operatorname{Spec}(T)$ such that $Q_{\alpha} \cap R=P_{\alpha}$ by [1, Theorem 5.10]. Since $T$ is UZD, by Proposition 1, $\bigcup_{\alpha \in \Lambda} Q_{\alpha}=\bigcup_{i=1} H_{i}$ for some $H_{i} \in \operatorname{Spec}(T)(i=1, \ldots, s)$. Now it follows that $\bigcup_{\alpha \in \Lambda} P_{\alpha}=\bigcup_{\alpha \in \Lambda}\left(Q_{\alpha} \cap R\right)=\bigcup_{i=1}^{\infty}\left(H_{i} \cap R\right)$. Hence $R$ is a UZD.

(ii) By hypothesis $B$ has only a finite number of minimal prime ideals, say $Q_{1}, \ldots, Q_{t}$. Notice that each $B / Q_{i}(i=1, \ldots, t)$ is a finite integral extension of $A /\left(Q_{i} \cap A\right)$ and $A /\left(Q_{i} \cap A\right)$ is a UZD (for $\left.i=1, \ldots, t\right)$. We prove that $B / Q_{i}$ is a UZD for each $i \in\{1, \ldots, t\}$. Then it will follow that $B$ is a UZD. Hence it suffices to prove (ii) in the case in which $B$ is an integral domain. Let $K$ denote the quotient field of $B$. Let $X$ be an indeterminate over $K$. Consider $V=K[[X]]=K+M$ where $M=X K[[X]]$. Let $B_{1}=B+M ; A_{1}=A+M$. Since $B$ is a finite integral extension of $A$, it follows that $B_{1}$ is a finite integral extension of $A_{1}$. As $A$ is a UZD, $A_{1}$ is a Z.D. ring by [13, Remark 2.1]. Hence $B_{1}$ is a Z.D. ring by [9, Theorem 2.9]. Again by [13, Remark 2.1], $B$ is a UZD. This completes the proof of (ii).

REMARK 13. We mention an example to show that Proposition 12 (ii) does not extend to infinite integral extensions. Gilmer and Huckaba in [8, Example p.211] have constructed for a fixed prime $p$ an infinite algebraic extension $L$ of the field of rationals $Q$ such that the integral closure $\overline{Z_{p}}$ of $Z_{p}$ in $L$ has an infinite number of maximal ideals. 
Since $Z_{p}$ is a 1 -dimensional quasilocal domain it is clear that $Z_{p}$ is a UZD. As $\overline{Z_{p}}$ has an infinite number of maximal ideals, $\overline{Z_{p}}$ is not a UZD.

We conclude this note with the following Proposition which determines when every overring of an integral domain is a UZD.

Proposition 14. Let $R$ be an integral domain with quotient field $K$. Then each overring of $R$ is a UZD if and only if the integral closure of $R$ in $K$ is a Prüfer domain with only finitely many maximal ideals.

Proof: $(\Rightarrow)$ Let $\bar{R}$ denote the integral closure of $R$ in $K$. Let $Q \in \operatorname{Spec}(R)$. Let $\alpha \in K, \alpha \neq 0$. Let $X$ be an indeterminate over $\bar{R}_{\mathbf{Q}}$. Let $g$ denote the $\bar{R}_{\mathbf{Q}}$ homomorphism from $\bar{R}_{Q}[X]$ to $\bar{R}_{Q}[\alpha]$ determined by $g(X)=\alpha$. Now $\bar{R}_{Q}[\alpha]$ is a UZD and hence it has only a finite number of maximal ideals. We assert that $\operatorname{ker} g \nsubseteq \mathbf{Q} \bar{R}_{\mathbf{Q}}[X]$. For if ker $g \subseteq Q \bar{R}_{Q}[X]$ then $\left(\bar{R}_{Q}[X]\right) /\left(Q \bar{R}_{Q}[X]\right) \simeq\left(\bar{R}_{Q}\right) /\left(Q \bar{R}_{Q}\right)[X]$ becomes a homomorphic image of $\bar{R}_{\mathbf{Q}}[\boldsymbol{\alpha}]$ which would force $\left(\bar{R}_{Q}\right) /\left(\bar{Q}_{Q}\right)[X]$ to have only a finite number of maximal ideals, a contradiction. Hence $\operatorname{ker} g \nsubseteq \mathrm{Q} \bar{R}_{\mathbf{Q}}[X]$. So by $[6$, Lemma 19.14] either $\alpha$ or $\alpha^{-1}$ is in $\bar{R}_{Q}$. Thus $\bar{R}_{Q}$ is a valuation ring for each $Q \in \operatorname{Spec}(\bar{R})$. Hence $\bar{R}$ is a Prüfer domain. Since $\bar{R}$ is a UZD, $\bar{R}$ has only a finite number of maximal ideals.

$(\Leftarrow)$ Let $A$ be any overring of $R$. Let $\bar{A}$ denote the integral closure of $A$ in $K$. Then $\bar{A}$ is a Prüfer domain with only a finite number of maximal ideals by [6, Theorem 26.1 (a) and Exercise 14, p. 331]. So $\bar{A}$ is a UZD by Proposition 4(ii). Now Proposition 12 (i) implies that $A$ is a UZD.

\section{REFERENCES}

[1] M.F. Atiyah and I.G. Macdonald, Introduction to commutative algebra (Addison Wesley, Reading, MA, 1969).

[2] N. Bourbaki, Commutative algebra (Addision Wesley, Reading, MA, 1972).

[3] P.M. Eakin, 'The converse to a well known theorem on Noetherian rings', Math. Ann. 177 (1968), 278-282.

[4] E.G. Evans, 'Zero divisors in Noetherian-like rings', Trans. Amer. Math. Soc. 155 (1971), 505-512.

[5] R. Gilmer, Multiplicative ideal theory 12, Queen's papers on Pure and Applied Mathematics (Queen's University, Kingston, Ontario, 1968).

[6] R. Gilmer, Multiplicative ideal theory (Marcel-Dekker, New York, 1972).

[7] R. Gilmer and W. Heinzer, 'The quotient field of an intersection of integral domains', $J$. Algebra 70 (1981), 238-249.

[8] R. Gilmer and J.A. Huckaba, 'The transform formula for ideals', J. Algebra 21 (1972), 191-215.

[9] W. Heinzer and D. Lantz, 'The Laskerian property in commutative rings', J. Algebra 72 (1981), 101-114. 
[10] W. Heinzer and D. Lantz, 'Commutative rings with ACC on n-generated ideals', J. Algebra 80 (1983), 261-278.

[11] I. Kaplansky, Commutative rings (University of Chicago Press, Chicago, 1974).

[12] J. Ohm and R. Pendleton, 'Rings with Noetherian spectrum', Duke Math. J. 35 (1968), 631-640.

[13] S. Visweswaran, 'Subrings of $K\left[y_{1}, \ldots, y_{t}\right]$ of the type $D+I$ ', J. Algebra 117 (1988), 374-389.

\section{Department of Mathematics}

Saurashtra University

Rajkot

India 360005 